\title{
A Multi-Objective Optimization Algorithm for Routing Path Selection and Wavelength Allocation for Dynamic WDM Network using MO-HLO
}

\author{
Hamsaveni M, Savita Choudhary
}

\begin{abstract}
The data transmission system in the optical WDM network increases the speed of packet transmission by the wavelength of light beams. The Selection of the wavelength and the shortest path to transmit the packets form source to destination is a challenge in a large network architecture. To solve these two problems, the optimization model must handle both the objectives. In this paper we are proposing a novel multi-objective optimization algorithm to solve both the problem of wavelength allocation and shortest path identification in a WDM network. This can be achieved by the enhanced model of Multi-Objective Hunger Locust Optimization algorithm (MO-HLO). In this, it analyse traffic level in a network path and the availability of wavelength present at each time instant. The proposed system retrieves the parameters of network architecture and with the weight value of dynamic traffic occur in the routing path. Among these data, the optimization selects the best among overall feature set of the WDM arrangement. The MO-HLO algorithm extracts the combination of each attribute to form the cluster that segregates the routing path along with the traffic range. From the fitness of the objective function of $\mathrm{MO}-\mathrm{HLO}$, the best routing path and the availability of wavelength for a node can be analysed at each time instant.
\end{abstract}

Index Terms: Wavelength Division Multiplexing (WDM), Optimal routing system, Multi-Objective Hunger Locust Optimization algorithm (MO-HLO).

Keywords: The proposed system retrieves the parameters of network architecture and with the weight value of dynamic traffic occur in the routing path.

\section{INTRODUCTION}

In the fast moving culture of the world technologies, the internet helps in large source of applications to process all the common functionality for the general usage in the human life. Without the internet facility no any applications can run in the standard condition. Due to the large demand in the usage of

Manuscript received on April 10, 2021.

Revised Manuscript received on May 26, 2021.

Manuscript published on June 30, 2021.

* Corresponding Author

Hamsaveni $\mathbf{M}^{*}$, Assistant Professor, Department Computer Science \& Engg., Vidyavardhaka College of Engineering, Mysuru, India. E-mail: hamsaveni.m@vvce.ac.in

Dr. Savita Choudhary, Associate Professor Department of Computer Science \& Engg, Sir MVIT, Bengaluru, India. E-mail: savitha cs@sirmvit.edu

(c) The Authors. Published by Blue Eyes Intelligence Engineering and Sciences Publication (BEIESP). This is an open access article under the CC BY-NC-ND license (http://creativecommons.org/licenses/by-nc-nd/4.0/) communication through the internet, the traffic in the network also getting increased. The system needs to consider and manage these type of issues to provide high speed network arrangement with the optimal scheduling method.

For the high speed internet connectivity, the light sources was used to modulate and transmit the data by utilizing the wavelength of light beams in Wavelength Division Multiplexing (WDM) system. This type of multiplexing technique achieved high speed data communication in a network area. Since, the bandwidth in the WDM has in the high range that enables the light in various wavelength according to the frequency of signal that is multiplexed in a same transmission medium. This also reduce the data loss compare to the other wired transmission channel.

In a large size of network area, the data signal transmission must be scheduled to prevent the data traffic which may cause loss of data in the channel. For this process, the routers are used to schedule and switch the line connections to manage the packet scheduling in the WDM network. This will also find the short path of data transmission to achieve the minimum travel time of data to reach the destination in a stochastic network region. To differentiate the data from one node to another node of system, the wavelength for individual system was varied and modulated with the data to represent the system ID. Since for the large network area, the standard wavelength cannot be used for these systems which results in collision in the data while at the multiplexing of these signals. To solve this problem, dynamic wavelength allocation technique was used to prevent the traffic level in a large network region.

From these two objectives of WDM system, there are several methods to perform the shortest path routing model and the optimal allocation of wavelength in a network. To achieve this, some methods implemented individual model to solve the problem in two objectives in WDM individually. This paper proposed a multi-objective optimization model to solve both the routing problem and optimal dynamic wavelength allocation in a network. This type of multi-objective optimization system performs effective routing system by considering both the objective which reduce the time complexity and overcome synchronization problem between the nodes to communicate each other. This type of optimal routing system can be validate by using the parameters such as blocking probability, throughput and other parameters to estimate the error rate in data transmission.

Published By:

Blue Eyes Intelligence Engineering and Sciences Publication

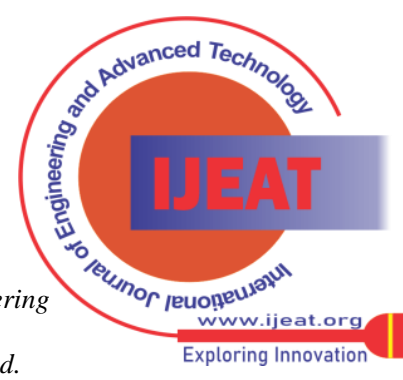


The main contribution of the proposed model is

1. To find the best routing path from the shortest distance and the weight value of traffic in the line between nodes.

2. To identify the optimal selection of best wavelength that matching for the node which is to transmit the data with minimal loss.

3. To achieve low blocking probability due to the high speed optimization model.

4. To reduce the size of iteration count that reach the less cost at smaller in value.

The descriptions about the paper algorithm and the validation of proposed model can be organized as following sections:

The section II presented the survey of various existing methods in WDM system. The proposed optimization method and its algorithm descriptions are discussed in section III. The result validation and the comparative study of proposed work over traditional routing system is described in the section IV. Then the discussion about the result analysis and the performance are discussed in section $\mathrm{V}$. the overall paper work is justified and concluded with future enhancement is discussed in section VI.

\section{RELATED WORK}

This section presented the survey of various methods in the routing model of WDM and also optimal selection of wavelength allocation problem with different optimization concept.

In [1], author proposed a multi-objective routing and wavelength allocation in WDM network using an enhanced model of Strength Pareto Evolutionary Algorithm (SPEA2). The main motivation of this method is to accept the maximum number of communication request with maximum number of wavelength allocation. This will reduce the blocking probability. Similarly in [2], author proposed a multi-objective optimization algorithm using hybrid evolutionary computation approach. This is to solve the problem of RWA in WDM network. Later, the Ant Colony optimization (ACO) algorithm was used in [3] to solve the RWA problem in WDM network. This is to estimate the dynamic anycast routing parameters for validating the traffic range in a network architecture while at communication. The Differential-Evaluation based Routing and Wavelength allocation (DE-RWA) was proposed in [4]. This can optimize both the network wavelength requirements and average path length in a network to find the optimal path allocation with best selection of wavelength from available list. In $[5,6]$, author review different types of RWA model in the large size of optical network and discussed about the performance of these methods. The main keywords that they have specified was light path, priority based ordering and wavelength allocation.

For the shortest path routing, the most general method for path identification is Dijkstra algorithm. This was also combined with several optimization algorithm to reduce the iteration count for large network architecture. For that, [7] representing the comparative analysis of Dijkstra algorithm with Ant Colony Optimization algorithm for a random or dynamic topology. In an elastic optical network, a constrained lower indexed block spectrum assignment policy was implemented in [8]. In the optical backbone networks, the
RWA problems were solved by analyzing different parameters in the network arrangement. By considering that, [9] analysed the energy and fatigue-aware RWA model that representing Energy and Fatigue Aware Heuristic with Unnecessary Reconfiguration Avoidance (EFAH-URA) to balance the three aspects in WDM network. Similarly in [10], author proposed an improved offline multi-objective routing system in WDM. In this a parallel hybrid algorithm flower pollination and intelligent water drop algorithm with simulated annealing (SA) (FPIWDSA) algorithm was proposed to perform parallel computation which can reduce the time complexity. In [11], a pre-trained network arrangement was proposed by using the reinforcement-learning-based RWA algorithm. This estimate the traffic distribution in a network that maximize the number of paths to be accommodated to a network. In [12], author proposed a cross-layer optimization framework for integrated optical switches in data centers. Using this framework, this can optimize four categories of integrated optical switches such as Crossbar, PILOSS, DRAGON and FODON.

In optimization algorithm, there are several methods are there to perform optimal selection of best solution among the evaluation of several parameters. In that, a novel evolutionary neural network with Locust Swarm Optimization (LSO) was proposed in $[13,14]$ for the application of intrusion detection in a network. This is to analyse the performance of LSO to select the best solution with increased speed of time. In [15, 16], papers proposed a machine learning based prediction model and Markov chain probability model to solve the RWA respectively. This type of optimal allocation can be applied in the massive MIMO-enabled 5G network in WDM. In [17], the network architecture collaborates the MIMO communication with WDM based fronthaul for multicast service beam forming model. This is to improve the speed of communication and increase the throughput of network. To improve the speed of optical network, [18] investigated about the hybrid optical amplifiers. This analysed different combination of amplifier design with erbium-doped fiber amplifier (EDFA) model. The paper in [19] also presented a survey of different machine learning algorithm to solve the problem of resource allocation. In [20], author proposed a novel model of routing and spectrum allocation algorithm for the elastic optical network arrangement. This type of optical network arrangement was implemented by Tradeoff-RnI algorithm that refers the tradeoff parameters between consumed resources and interval with the boundary of it. In a mixed grid based optical network, [21] proposed a service differentiation based spectrum defragmentation to solve the issue in flexible grid system. In [22], a novel heuristic method for lightpath routing and wavelength allocation was proposed, this heuristic algorithm is based on the concept of wardrop equilibrium that is to adaptively equalize the mean delay

From these survey statements, there are two different objectives are considered in the WDM system to solve it for better data transmission system. Blue Eyes Intelligence Engineering and Sciences Publication

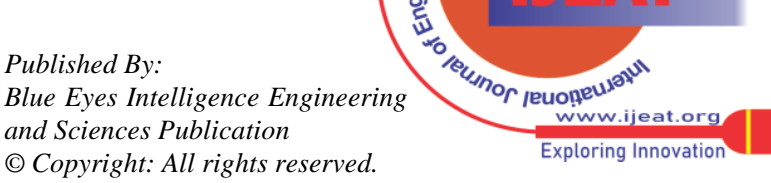


The best routing path selection and the dynamic wavelength allocation are the major key points that are focused in the proposed work. According to the review of various methods, it is better to implement a multi-objective optimization model to solve both of the problem in WDM system.

For enhancing the speed of optimization can be improve by implementing the concept of Locust search method that predicts the best location to get the food particles and eat well. Whereas, the Hungry Locust consume more food compare to the general locust particles. In this paper work, the Multi-Objective Hungry Locust Optimization (MO-HLO) algorithm is used to enhance the performance of routing path and optimal wavelength selection in WDM. The detailed description of the proposed method and its algorithm explanations are in the following sections.

\section{PROPOSED WORK}

The paper work proposed a novel optimization algorithm to solve the multiple objective problem in WDM network. In a WDM network, the speed of data transmission is depends on the availability of network path from the source to the destination. In general, the routing path is processed to select the shortest path by using several routing shortest path analysis method to process the data communication through the network medium. Since, for every networking system in WDM, there will be an upper limit for the wavelength usage for data transmission. This identifies whether the wavelength for a node can be suitable for conversion to the node. If it satisfies, then this will be consider as the optimal solution for wavelength allocation for a node in a network. The routing problem is in the same condition as in the traditional switching process of a channel. But the limiting factor for it is the number of channels present in the network. Here the traffic in the network is considered as the dynamic random form that varied the value at each time instant. These parameters are considered in the optimization problem to extract the property value at each iteration. This was updated by varying the value weight value and the position of particles in the overall coverage area.

The merits of proposed work is

1. The major advantage of the proposed work it that it can manage both the routing process and the optimal wavelength selection problem to form as Multi-Objective optimization.

2. This can solve the issue of random dynamic traffic range in the network path that is to select the best path according to the weight value of it.

3. Due to the multi-objective optimization method of routing and wavelength allocation, it improves the speed of data transmission along with the multiplexed signal over the channel.

4. This also reduce the blocking probability that indicates that the waiting time for the nodes in the network was reduced compare to traditional model.

5. The result can improve the Quality of Service (QoS) which increase the performance of WDM.

The figure 1 shows the overall flow diagram of proposed optimization method. In that, it shows the update of the particles is based on the weight value of traffic in the routing path and the node properties which is suitable for wavelength conversion for data communication in the channel. From the wavelength pool, it identifies the availability of wavelength for the arrived node and find the best suitable wavelength for the node to reduce the large usage of bandwidth in the network.

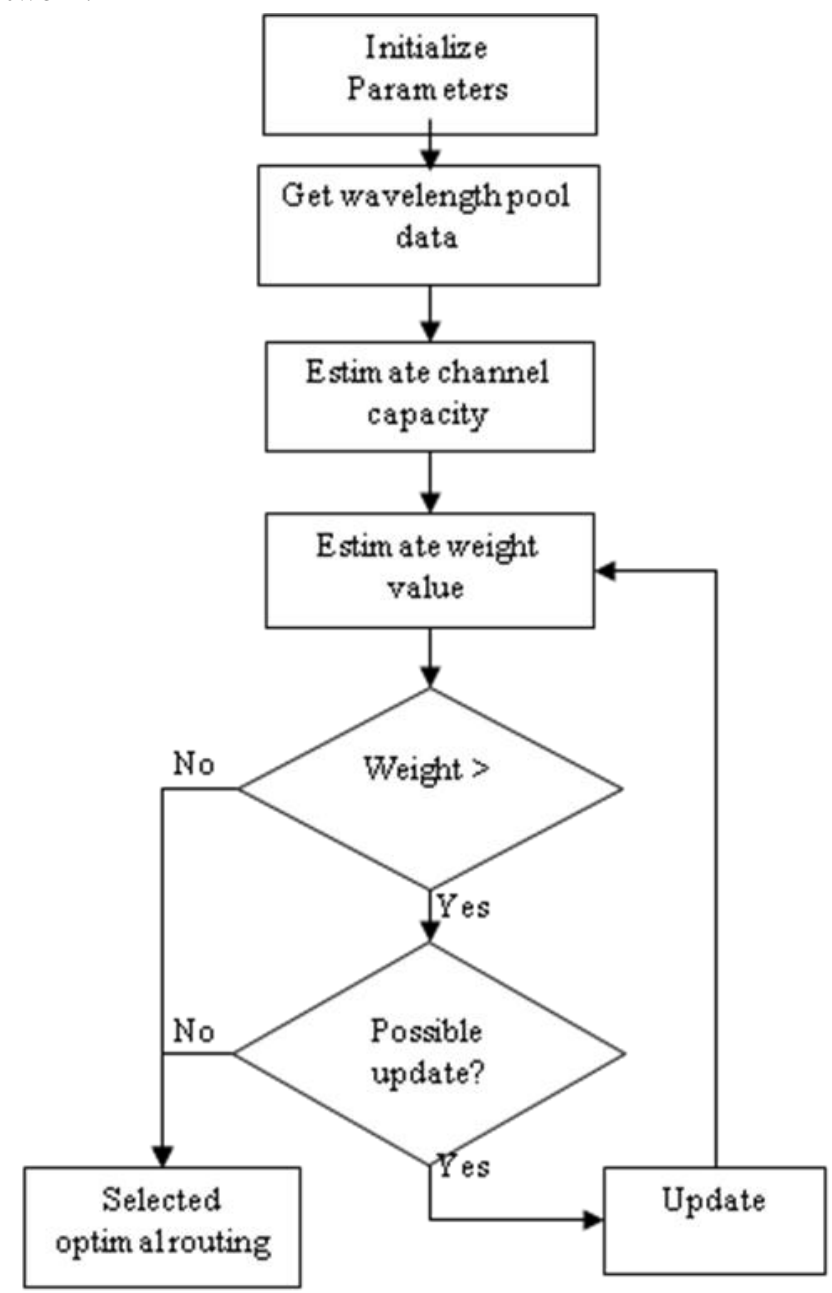

Figure 1: Overall flow diagram of proposed MO$\mathrm{HLO}$

The detailed explanation about the conventional routing process with the conventional wavelength selection and the proposed MO-HLO algorithm are classified in the following subsections as,
a) Path Routing Model,
b) Wavelength allocation,
c) MO-HLO algorithm.

\section{A. Path Routing Model}

In the network routing model, there are three common design was addressed in the routing assignment problem in WDM system.

These can be classified as fixed routing, fixed-alternate routing and adaptive routing model.

Published By: Blue Eyes Intelligence Engineering and Sciences Publication

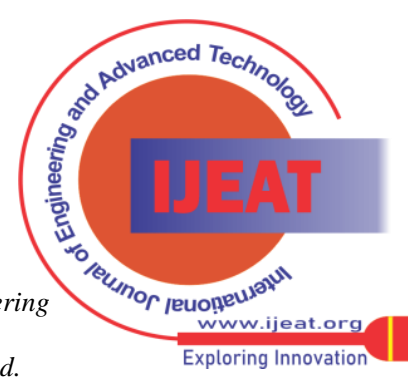




\section{A Multi-Objective Optimization Algorithm for Routing Path Selection and Wavelength Allocation for Dynamic WDM Network using MO-HLO}

Fixed routing model: In this type of fixed routing model, the router was placed in the fixed for the each source to destination pair of connectivity.

If there is any request passed

by the source node, then it will establish the path in fixed routing table. This is simple to implement and cost effective. Since, it is the fixed arrangement, the maintenance of global network state information is not required. There will be a pre-determined routing table was used to identify the path for the specified source and destination node in a network.

In this, if there is no any common availability of wavelength for every link in the routing path, then the connections will be blocked. This type of fixed routing path network has very less option for the routing process which may cause high level of blocking in a network arrangement.

Fixed-Alternate Routing: This type of fixed-alternate routing model, it requires to maintain a routing table for each node in a network. This contains the data which contains the list of fixed routes for each destination node ordered in the basis of number of links that is segmented to the destination. According to the process of arrangement in the routing table the shortest path was ordered as the top most layer in the routing table. Among these, if there are any same weight value in the routing table, then this was selected any one of the link based on the random identification of network path. If there is any connection request passed by the source node, then it find the connection on each routing tables in the nodes until it satisfy the matched wavelength found for the communication. If there is no any available wavelength was found in the alternate routing tables, then the connection was blocked and it may loss the link. Since, this is the enhanced model of fixed routing which can reduce the blocking probability of connection loss compare to the fixed routing concept.

Adaptive Routing model: This type of routing model is a dynamic connection establishing that links the destination node according to the state of network arrangement. This updates the routing path based on the availability of nodes and its weight value of the link. These form of routing allocation and the routing path identification was processed by using the adaptive routing algorithms to solve the shortest path identification problem. This selects the optimal routing path according to the availability of link and also based on the busy state of nodes. Since, in this routing process, the routing table in the destination nodes may change dynamically at each time instant. Due to this arrangement, the connection will confirm for a node that is passed the connection request which results in reduced amount of blocking probability value compare to the previous fixed routing mode.

\section{B. Wavelength allocation}

In the wavelength allocation process, there may be two different way to present best wavelength to the source and destination. In that one is based on the same wavelength is allocated for the overall network that spans around the source to the destination node. Another one model is to use the different wavelength that are multiplexed in a same channel of WDM. There are several dynamic wavelength allocation algorithms were implemented in various application whish are listed as

- Random (R) Wavelength allocation,

- First-fit (FF) allocation,

- Most-Used (MU) allocation,

- Least-Used (LU). method of wavelength allocation.

\section{Proposed MO-HLO algorithm}

For iter $=1$ to $\mathrm{m}$ be represent as

$y=\left\{N_{s} L\right\}$

For $\mathrm{i}=1$ to $\mathrm{n} / /$ 'n' is node count

Update $\gamma_{i_{j j}}^{k}$ by the equation (1)

equation (2). point for identifying path length vector between nodes.

wavelength among available wavelength value in table

Else

Acknowledge to source

End If

End For ' $j$ '

End For ' $i$ '

End For

in the dynamic network topology. In the traditional model of wavelength allocation process, the heuristic algorithms were used to find the availability of wavelength when there is any request passed for connecting nodes. There are several policies to allocate the optimal wavelength for the nodes for connection process. In that some methods will share the wavelength completely and some follows partitioning of wavelength to reuse the same wavelength for various connection problem. Among that, the dynamic wavelength allocation system can perform better and optimal wavelength allocation with reduces blocking probability than the other

In this section, the detailed steps of proposed MO-HLO algorithm for routing and wavelength allocation was described. The algorithm 1 describes the step by step procedure for proposed MO-HLO algorithm for Routing and Wavelength allocation in WDM network.

\section{Algorithm 1: MO-HLO algorithm for RWA}

Input: Network table $\left\{N_{i}\right\}$, flow link cost $c_{i j}$

Output: Best routing path and wavelength selection

Initialize $y$ be the weight value of network link which can

For $\mathrm{j}=1$ to $l / /$ ' $l$ ' is the number of links

Generate link flow $\left\{f_{i j}^{k}\right\}$ for ' $k$ ' number of trials

Compute route choice probabilities $\left\{\int_{i j}^{k}\right\}$ as in

Update flow pattern, $f_{i j}^{k+1}$ using equation (3)

Check convergence for each $\mathrm{k}+1$ value

Calculate $G(y)=\max \left(y_{i j}^{k_{j}}\right)$. // Find highest possible

Calculate $L(j)$ from equation (4) // Find the distance

If free $\lambda$ available in flow link, then

Calculate $\Delta_{\mathrm{p} p}$ using equation (5) // Find optimal

Calculate $c_{\mathrm{f}, \mathrm{f}}$ using equation (6) // Update the cost

Choose the shortest path from the updated table ' $y_{i, j}^{k}$ '

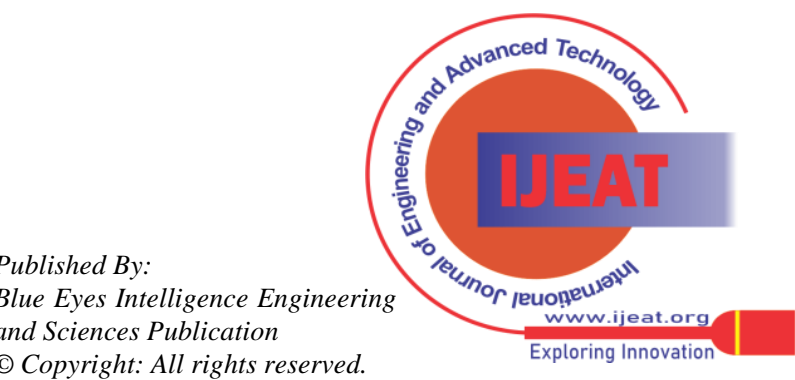


In this optimization process, the particles are initialized for the network parameters of connective link and the random traffic in the routing path. The concept of HLO algorithm is that the Locust particles find the distance that can cover the food particles present in the area to travel and consume more. Since the hunger Locust can travel large area in short duration that optimally find the best location of food particles. The reproductive

process also make fast to update the Locust group strength to form it as a cluster of family. By using the advantages of these hunger locust, the proposed algorithm formed the routing process by selecting the best path to get the connectivity speed compare to the other routing method. While routing the shortest path, this also identify the available wavelength for the source node with its properties to provide the suitable range of wavelength to transmit the data in the WDM network.

Let the ' $\lambda$ ' is defined as the wavelength that are initialized in the network and ' $Y$ ' representing the traffic weight in the node link. The network was initialized for ' $N$ ' set of nodes for the ' $L$ ' number of fiber links between each nodes. These parameters are extracted to generate the initial particles of locust to move over the network.

According to these, the weight value of the link can be update for the node to node communication can be estimate by using the equation (1).

$\gamma_{i, j}^{k}=\gamma_{i, j}\left(f_{i, j}^{k}\right), \forall(i, j) \in L$

From this link weight value the probability of the routing choice for the link cab ne represent as $\left\{i_{i j}^{k}\right\}$. This can be calculate by using the equation (2).

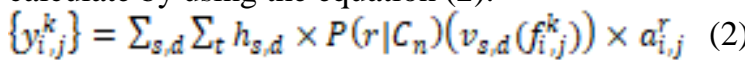

Where, $(s, d)$ represent the source to destination pair.

$v_{s d}$ - speed of packet transmission from source to destination.

$\sigma_{i, j}^{r}$-represents the number of times a link in (i,j) appears in a switch ' $r$ '.

From the detected router path, the data flow pattern can be represent as $f_{i j j}^{k}$ for $k$ number of trials. The flow rate for each selection of node and its link will be update for each iteration to validate the convergence of data loss for the selected routing path. This was extracted and found the minimum loss of path that can be selected as the routing path for that node. This can be evaluate by the equation (3).

$f_{i j}^{k+1}=f_{i j}^{k}+\alpha_{n} \times\left(j_{i j}^{k}-f_{i j}^{k}\right)$

From this the $G(y)$ represents the highest possibility of selecting the length of routing path for a node. This highest length indicates the maximum area that it covers the network for that particulate iteration. From this, the distance matrix for the $j$ th link can be evaluate by using equation (4).

$L(j)=\frac{1}{n} \sum_{x=1}^{n}\left\|N\left(f_{i_{j}}^{k}\right)-G(y)\right\|$

The delta difference for each link represent the available wavelength that is matched and optimally selected in equation (5). In that, it validates the optimal matching point for more than $90 \%$ of channel capacity to provide better data transmission with less amount of packet loss.

$\Delta_{i j}=0.9 \times \Delta_{j-1}+\mu \times \partial L / \partial W_{i}^{\mathrm{l}}$

The cost value is depends on the weight value updated for each iteration and this can be evaluate by the equation (6). $c_{i, j}=W_{j}^{l}+\Delta_{i, j}$
Where, $\mathrm{k}$ - number of trials for a node.

The cost value will reduce for the network arrangement for each iteration which is based on the convergence curve by optimally selection of best routing path and wavelength availability.

This forms the overall process of routing path selection and wavelength selection for the node communication by using MO-HLO optimization algorithm. For every iteration count, this refers the distance between the links to reach the destination with minimum trials. In parallel, it also identifies the available wavelength to provide optimal selection of both routing path and wavelength with minimum cost of network link from source to destination. This improves the speed of network connectivity and the optimal selection of wavelength increases the speed of data transmission with reduced probability of stop and wait process.

\section{RESULT ANALYSIS}

This section describes about the validation of result from the proposed optimization algorithm that is presented in the WDM application. For this analysis part, the functions for the algorithm was developed in the Python simulation platform which is modeled and executed with the commonly available network topology of WDM. In that data, the network parameters and the initial values of traffic level in the routing path were initialized and validated. This section also shows the performance of proposed RWA optimization model by estimating the comparative parameters to represent the efficiency of MO-HLO algorithm compared with other existing RWA model.

The proposed work of optimal RWA system can be compare and justify the result with an existing system of AWRA, HRA and HWRA in [23] for the standard network topology of 20 Nodes and 38 link based random network architecture. Since, the topology that is followed in the comparison is random formation, this can be justified as that the proposed algorithm can solve the problem in dynamic network architecture.

The parameters that are considered for the validation of validation of proposed method are Blocking probability, throughput, time delay, and Quality of Service (QoS) that are can be represented and calculated as

$B P=\frac{R C}{T C}$

Where, BP - Blocking probability,

$\mathrm{RC}$ - Rejected Connection

TC - Total Connection

$\Delta Q o S(\%)=\frac{B P_{1}-B P_{2}}{1-B P_{1}} \times 100$

Where, $B P_{1}$ and $B P_{2}$ are the blocking probability for the change in wavelength availability from 10 to 20 per units.

The bit error rate can be calculated by using the equation (9).

$$
B E R=\frac{N_{\text {Error }}}{N_{\text {Total }}}
$$

Published By: Blue Eyes Intelligence Engineering and Sciences Publication

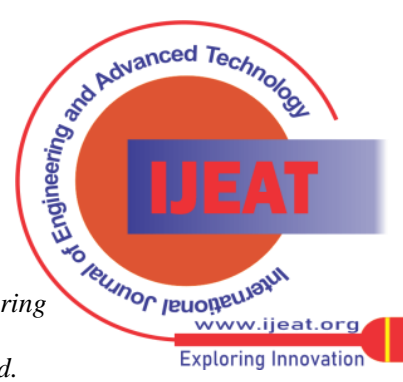


Where, $N_{\text {Error }}$ - Number of bits in error at data transmission.

$N_{\text {Total }}$ - Total number of bits that are allocated for data transmission.

The blocking probability of a method can be represent as the ratio between number of rejected

connection by the nodes and

total number of available connections in a network. Since, the rejected connection is can be estimate from the connections that are timed out from the communication due to unavailability of wavelength or by the routing time based on the traffic in a network path.

The change in Quality of Service (QoS) of a network RWA allocation can be evaluated based on the blocking probability of the network that changes from one wavelength value to another wavelength for improving the speed of communication and routing capacity. This defines the ratio of difference between the one wavelength parameter to another wavelength parameter to the unit difference of initial wavelength. This can be represent in percentage of value.

The figure 2 shows the network structure of random formation of 20 nodes and 38 links WDM arrangement. That is referred from [23]. In this, the blue circle represents the nodes in the network and the line that interconnecting the nodes are representing the fiber cables in WDM architecture.

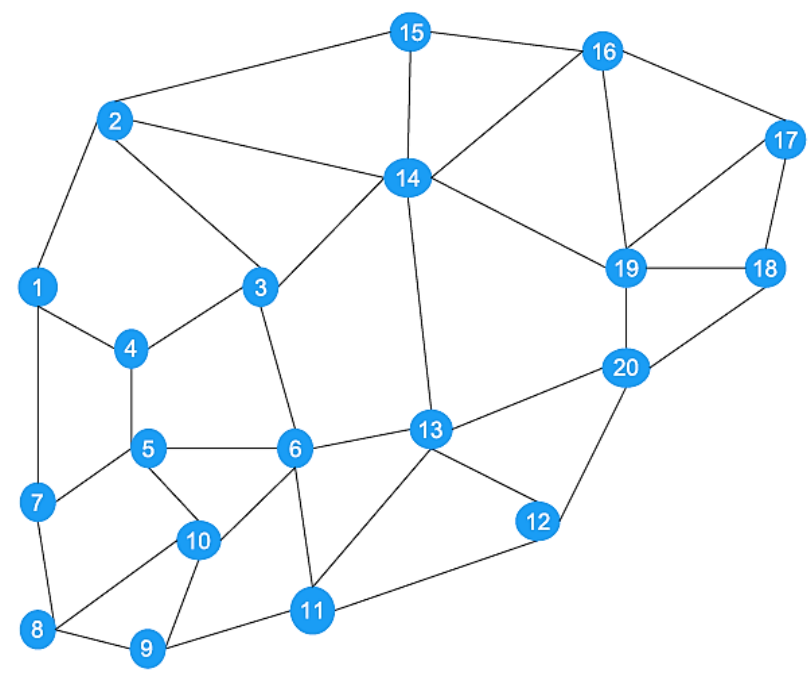

Figure 2: Testing Network Architecture of WDM

Figure 3: Comparison result of Blocking Probability

From this network arrangement, the blocking probability for the proposed work compares with the existing methods of AWRA, HRA and HWRA is shown in the figure 3 separated by color code for each method. In that, the $\mathrm{x}$-axis of the graph representing the traffic level from the range of 40 to 110 to validate the performance of proposed method. The $y$-axis of the graph representing the blocking probability ratio value. The graph update for blocking probability changes for each traffic level in the network. According to the standard network characteristics, the blocking probability may increase with the increase in traffic value.

From the graph it shows the trend line of blocking probability that it represents that proposed MO-HLO algorithm achieved better results of reduced $\mathrm{BP}$ value compare to other existing methods of RWA. According to the $\mathrm{BP}$ value, for the number of available wavelength, it can improve the Quality of Service (QoS) for the network.

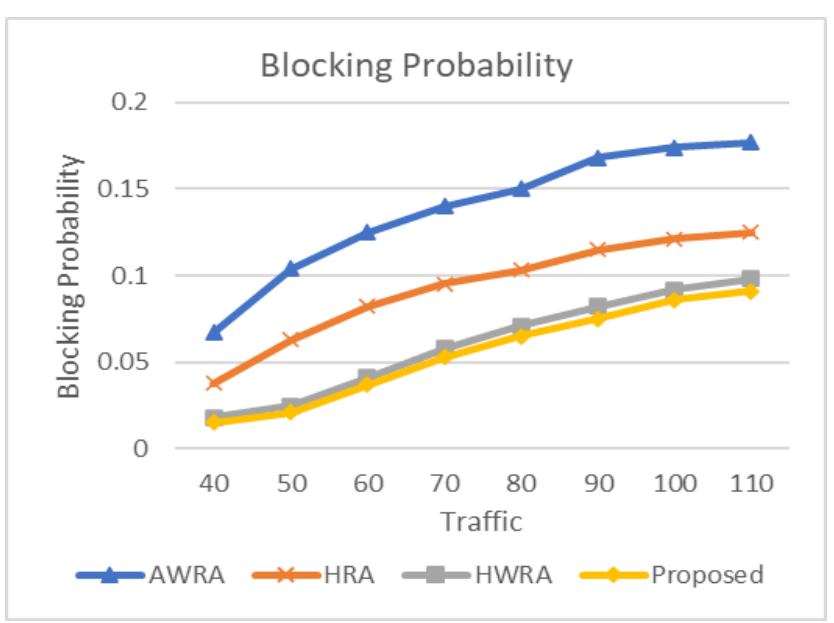

Figure 4: Comparison result of Blocking probability

To validate the QoS of the WDM network, then this test was fine-tuned by making the change of number of wavelengths in a network in the difference of 10 to 20 in count of values. As per the equation (8), the BP for one wavelength set and BP for another wavelength set are estimated and represented as BP1 and BP2 respectively. According to that, the change of QoS (\%) was estimated and compared with the existing work that is shown in the figure 5 . Here, the trend line of graph shows that he proposed work achieved higher the percentage of QoS compare to other existing methods.

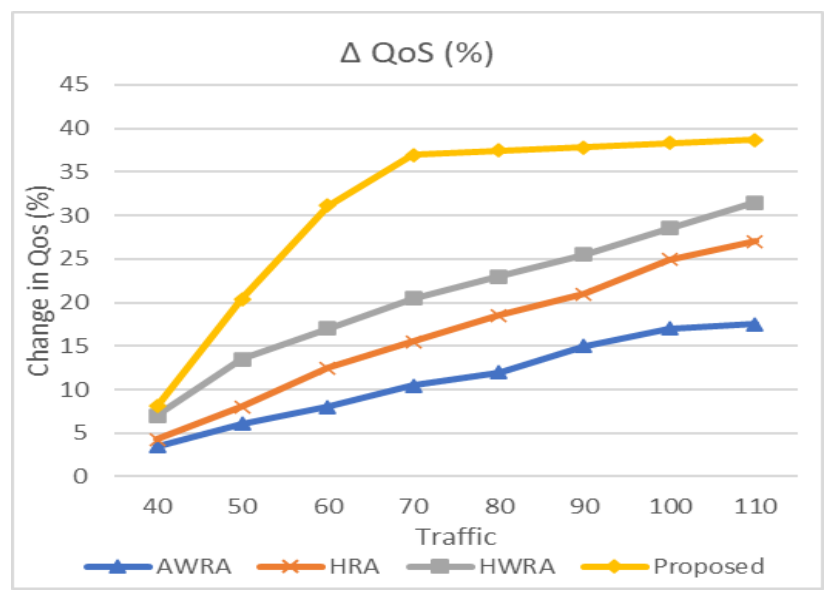

Figure 5: Comparison result of Change in QoS (\%)

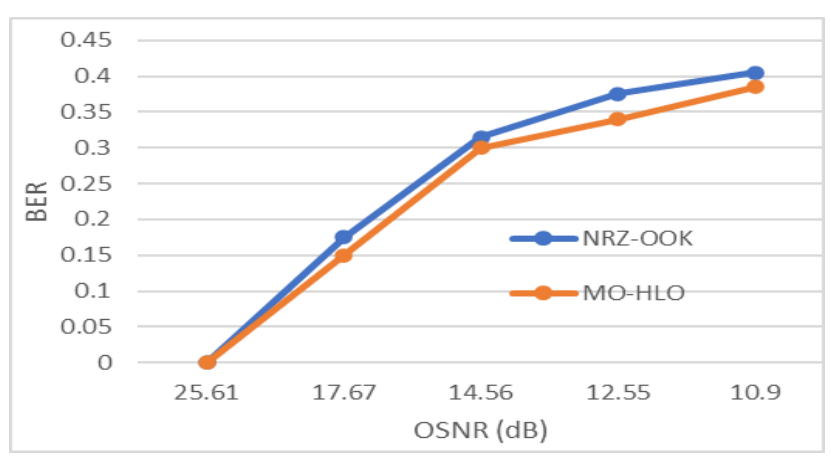

Figure 6: Comparison result of BER

Published By:

Blue Eyes Intelligence Engineering and Sciences Publication

(C) Copyright: All rights reserved.

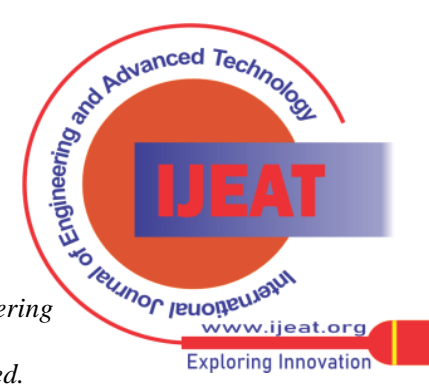


To evaluate the BER of proposed RWA method, this was tested in the IndiaNet Topology that is referred from [24]. The figure 6 shows the Bit Error Rate (BER) comparison of proposed method with NRZ-OOK algorithm. Also, this was tested in the COST 239 topology (11 nodes and 52 links) [25] based WDM network architecture. From this the wavelength link usage was estimated and presented with the comparison of existing system. In that graph, the Design 1 and 2 are the existing work of [24]. The wavelength link usage represents the amount of wavelength that randomly selects with different trial for various traffic index. The figure 7 shows the comparison chart of wavelength link usage for different traffic index.

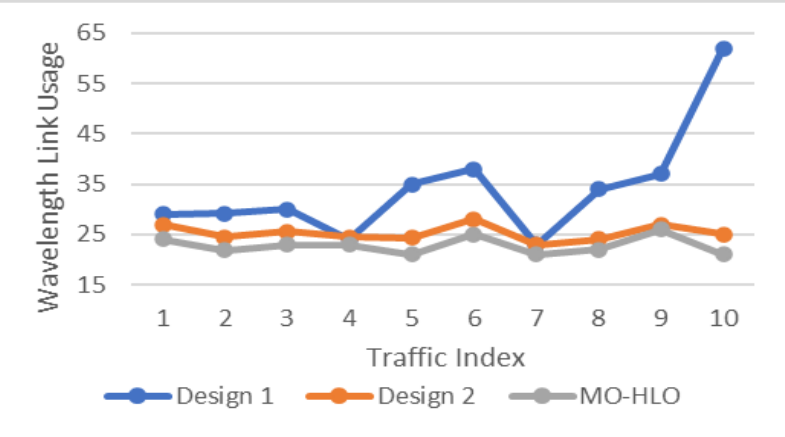

Figure 7: Comparison result of wavelength link usage for various traffic index

Figure 8 and 9 shows the graph plot for the parameters of various traffic density range and with the blocking probability analysis respectively. From this test analysis with different parameters it justifies that the proposed MO-HLO algorithm can solve the RWA problem in WDM network with high traffic level in the routing path.

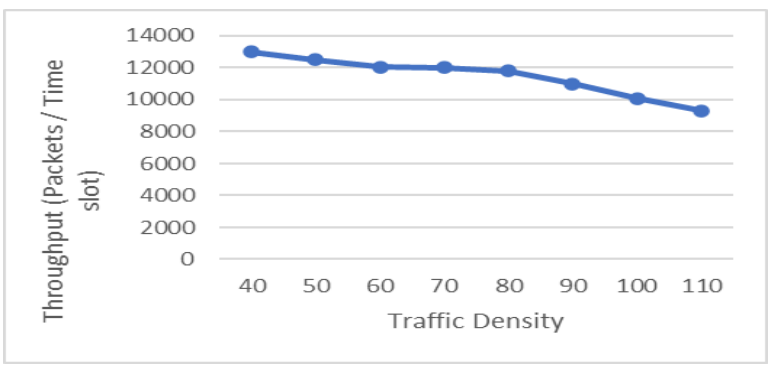

Figure 8: Traffic Density vs throughput

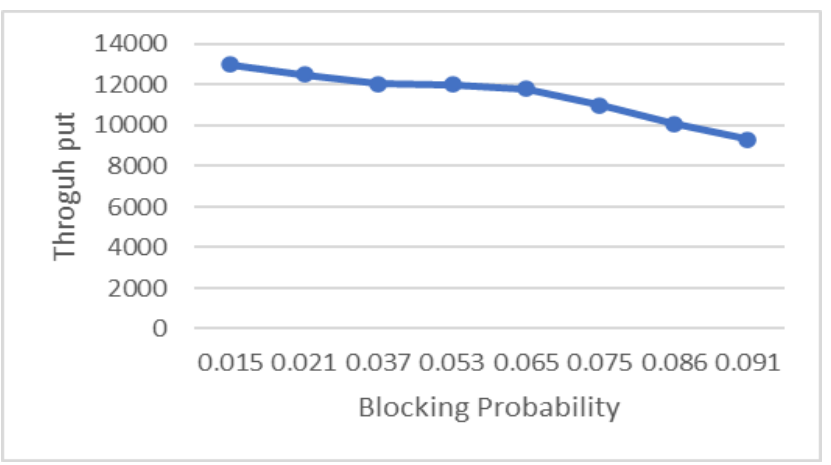

Figure 9: Blocking probability vs throughput

\section{DISCUSSION}

This section discussed about the performance result of proposed work in the optimal solution for RWA in WDM network. The validation of parameters compares the existing system of RWA shows that MO-HLO improves the range of communication with fast data transmission in the WDM network than the other state-of-art methods. This also reduce the complexity of a system represented by the reduction of number of iteration to reduce the cost value and to converge the error rate for each update of traffic level in the link path of nodes.

Generally, the complexity of the proposed system refers the time complexity to indicate the speed of performance. This time complexity for the proposed MO-HLO optimization algorithm can be represent by the notation as $O(q \times \ln (p))$ iteration cycles that required to find the matching of node attributes and for wavelength selection and ' $p$ ' be the predicted time taken for each iteration count. According to this description, the complexity is depends on the number of iteration and time taken for one iteration count to search for the best wavelength availability and routing path for a node to reach destination.

From the result analysis, the blocking probability was reduced in the percentage value of $\sim 2$ to $3 \%$ than the existing methods of RWA. Due to the optimal selection of wavelength for the node, the BER of data transmission was reduced with higher in speed of data transmission.

\section{CONCLUSION}

This paper proposed a novel optimization algorithm to manage the efficiency of WDM system by using Multi-Objective Hunger Locust Optimization (MO-HLO) algorithm. This analyse the properties of network that is to perform the identification of shortest path between the source and destination to transmit the data in high speed according to the verification of traffic condition in the network path. This also perform the optimal selection of best and suitable wavelength for the node according to the properties of the node that is to transmit the data.

The best selection of wavelength is detected for the node based on the properties of data with its node capacity. By these arrangement and optimal model of the WDM system, the blocking probability of the network communication gets reduced comparing to the other existing system. This also improve the speed of communication results in increase the throughput value that transmit more number of data packets in short duration of time. The proposed optimization algorithm reduced the time complexity and also reduce the data packet loss compare to traditional method of routing and wavelength allocation in WDM system.

\section{REFERENCES}

1. Leesutthipornchai, Pakorn, Naruemon Wattanapongsakorn, and Chalermpol Charnsripinyo. "Multi-objective routing wavelength assignment in WDM network using SPEA2 approach." 2009 9th International Symposium on Communications and Information Technology. IEEE, 2009.

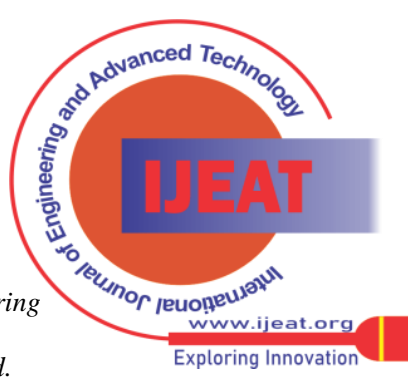




\section{A Multi-Objective Optimization Algorithm for Routing Path Selection and Wavelength Allocation for Dynamic WDM Network using MO-HLO}

2. Leesutthipornchai, Pakorn, Chalermpol Charnsripinyo, and Naruemon Wattanapongsakorn. "Solving multi-objective routing and wavelength assignment in WDM network using hybrid evolutionary computation approach." Computer Communications 33.18 (2010): 2246-2259.

3. Bhaskaran, Kavitha, Joan Triay, and Vinod M. Vokkarane. "Dynamic anycast routing and wavelength assignment in WDM networks using ant colony optimization (ACO)." 2011 IEEE International Conference on Communications (ICC). IEEE, 2011.

4. Lezama, Fernando, Gerardo Castañón, and Ana Maria Sarmiento. "Routing and wavelength assignment in all optical networks using differential evolution optimization." Photonic Network Communications 26.2-3 (2013): 103-119.

5. Chatterjee, Bijoy Chand, Nityananda Sarma, and Partha Pratim Sahu. "Review and performance analysis on routing and wavelength assignment approaches for optical networks." IETE Technical Review 30.1 (2013): 12-23.

6. Koganti, Ravi Teja, and Deepinder Sidhu. "Analysis of routing and wavelength assignment in large WDM networks." Procedia Computer Science 34 (2014): 71-78.

7. Sharan, Preeta. "COMPARISON OF DIJKSTRA ALGORITHM WITH ANT COLONY OPTIMIZATION ALGORITHM USING RANDOM TOPOLOGY IN ALL-OPTICAL NETWORK USING RWA." International Journal of Research in Engineering and Technology 04 (2015): 66-69.

8. Yuan, Junling, et al. "A constrained-lower-indexed-block spectrum assignment policy in elastic optical networks." Optical Switching and Networking 33 (2019): 25-33.

9. Natalino, Carlos, et al. "Energy-and fatigue-aware RWA in optical backbone networks." Optical Switching and Networking 31 (2019): 193-201.

10. Kaur, Harpreet, and Munish Rattan. "Improved offline multi-objective routing and wavelength assignment in optical networks." Frontiers of Optoelectronics 12.4 (2019): 433-444.

11. Shiraki, Ryuta, et al. "Dynamic control of transparent optical networks with adaptive state-value assessment enabled by reinforcement learning." 2019 21st International Conference on Transparent Optical Networks (ICTON). IEEE, 2019.

12. Wang, Zhifei, et al. "A Cross-Layer Optimization Framework for Integrated Optical Switches in Data Centers." IEEE Transactions on Computer-Aided Design of Integrated Circuits and Systems 39.3 (2019): 640-653.

13. Benmessahel, Ilyas, et al. "A new evolutionary neural networks based on intrusion detection systems using locust swarm optimization." Evolutionary Intelligence 12.2 (2019): 131-146.

14. Cuevas, Erik, Fernando Fausto, and Adrián González. "The Locust Swarm Optimization Algorithm." New Advancements in Swarm Algorithms: Operators and Applications. Springer, Cham, 2020. 139-159.

15. Martín, Ignacio, et al. "Machine learning-based routing and wavelength assignment in software-defined optical networks." IEEE Transactions on Network and Service Management 16.3 (2019): 871-883.

16. Truong-Huu, Tram, Purnima Murali Mohan, and Mohan Gurusamy. "Virtual Network Embedding in Ring Optical Data Centers Using Markov Chain Probability Model." IEEE Transactions on Network and Service Management 16.4 (2019): 1724-1738.

17. Xiao, Yuming, Jiawei Zhang, and Yuefeng Ji. "Integrated resource optimization with WDM-based fronthaul for multicast-service beam-forming in massive MIMO-enabled 5G networks." Photonic Network Communications 37.3 (2019): 349-360.

18. Wason, Amit, and Deepak Malik. "Performance investigation of hybrid optical amplifiers for high-speed optical networks." Journal of Optics 49.3 (2020): 298-304.

19. Gu, Rentao, Zeyuan Yang, and Yuefeng Ji. "Machine learning for intelligent optical networks: A comprehensive survey." Journal of Network and Computer Applications 157 (2020): 102576.

20. $\mathrm{Li}, \mathrm{Li}$, and Hong-jie Li. "Performance analysis of novel routing and spectrum allocation algorithm in elastic optical networks." Optik (2020): 164688.

21. Li, Yajie, et al. "Service differentiation-based spectrum defragmentation in mixed grid optical networks." Optical Fiber Technology 56 (2020): 102207.

22. Nath, Pantha Kanti, and Tamarapalli Venkatesh. "Lightpath routing and wavelength assignment for static demand in translucent optical networks." Photonic Network Communications (2020): 1-17.

23. Maurya, Rakesh Kumar, Jaisingh Thangaraj, and Vishnu Priye. "Dynamic Routing and Wavelength Assignment Using Cost Based

Published By:

Blue Eyes Intelligence Engineering and Sciences Publication
Heuristics in WDM Optical Networks." Wireless Personal Communications (2020): 1-22.

24. Gonsalis, Sting Salvador, and C. L. Triveni. "A Novel Approach for Static RWA based PLI Evaluation of WDM Networks in" WDM-NetSoft"." 2019 1st International Conference on Advances in Information Technology (ICAIT). IEEE, 2019.

25. Hai, Dao Thanh. "A Novel Integer Linear Programming Formulation for Designing Transparent WDM Optical Core Networks." 2019 International Conference on Advanced Technologies for Communications (ATC). IEEE, 2019.

\section{AUTHORS PROFILE}

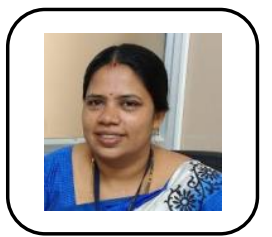

(s) a part-time research Scholar doing her $\mathrm{PhD}$ at Dept of CSE, Sir MVIT Bengaluru ,She has obtained her M.tech from VTU, Karnataka. She is working as a Assistant Professor at Dept. of CSE, Vidyavardhaka College of Engg. Mysuru.

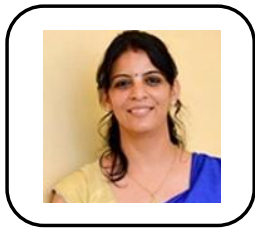

Dr. Savita Choudhary, working as a Associate. Professor at Dept. of CSE, Sir MVIT, Bengaluru. She has obtained her $\mathrm{PhD}$ from Bhagwanth University, Ajmer. She has expertise in the area of soft computing, Dr.Savita has published papers in several International, National conferences and journals, she has also guided many research 